\title{
Associações entre discriminadores do Sistema de Triagem de Manchester e diagnósticos de enfermagem
}

\author{
Association between Manchester Triage System discriminators and nursing diagnoses \\ Asociación entre el Sistema de Triage Manchester y el diagnóstico de enfermería
}

\author{
Betina Franco ${ }^{a, b}$ \\ Lurdes Busin ${ }^{\mathrm{a}, \mathrm{b}}$ \\ Tânia Couto Machado Chiancac \\ Vítor Monteiro Moraes ${ }^{\mathrm{a}, \mathrm{b}}$ \\ Ananda Ughini Bertoldo Pires ${ }^{a, b}$ \\ Amália de Fátima Lucena ${ }^{a, b}$
}

\section{Como citar este artigo:}

Franco B, Busin L, Chianca TCM, Moraes

VM, Pires AUB, Lucena AF. Associaçōes entre discriminadores do Sistema de Triagem de Manchester e diagnósticos de enfermagem. Rev Gaúcha Enferm. 2018;39:e2017-0131. doi: https://doi. org/10.1590/1983-1447.2018.20170131. aniversidade Federal do Rio Grande do Sul (UFRGS), Escola de Enfermagem. Porto Alegre, Rio Grande do Sul, Brasil.

${ }^{-}$Hospital de Clínicas de Porto Alegre (HCPA). Porto Alegre, Rio Grande do Sul, Brasil.

' Universidade Federal de Minas Gerais (UFMG), Escola de Enfermagem, Departamento de Enfermagem Básica. Belo Horizonte, Minas Gerais, Brasil.

\section{RESUMO}

Objetivo: Analisar associações entre discriminadores do Sistema de Triagem de Manchester e Diagnósticos de Enfermagem em pacientes adultos, classificados com prioridade clínica I (emergência) e ll (muito urgente).

Método: Estudo transversal realizado na unidade de emergência do sul do Brasil, entre abril e agosto de 2014. Amostra de 219 pacientes. Os dados foram coletados no prontuário online e analisados estatisticamente, com teste exato de Fisher ou qui-quadrado. Resultados: Encontrou-se 16 discriminadores e 14 diagnósticos de enfermagem. Houve associação entre sete discriminadores e cinco diagnósticos de enfermagem do tipo foco no problema, dentre Dor precordial ou cardíaca com o diagnóstico Dor aguda. Também houve associação entre três discriminadores com quatro diagnósticos de enfermagem de risco, dentre Déficit neurológico agudo com o diagnóstico Risco de perfusão tissular cerebral ineficaz.

Conclusão: Existem associações significativas entre discriminadores do Sistema de Triagem de Manchester e diagnósticos de enfermagem mais frequentemente estabelecidos na Unidade de Emergência.

Palavras-chave: Enfermagem em emergência. Triagem. Processo de enfermagem. Diagnóstico de enfermagem. Cuidados de enfermagem. Terminologia.

\section{ABSTRACT}

Objective: To analyze the association between Manchester Triage System flowchart discriminators and nursing diagnoses in adult patients classified as clinical priority I (emergency) and II (very urgent).

Method: Cross-sectional study conducted in an emergency department in southern Brazil between April and August 2014. The sample included 219 patients. Data were collected from online patient medical records and data analysis was performed using Fisher's exact test or the chi-square test.

Results: 16 discriminators and 14 nursing diagnoses were identified. Associations were found between seven discriminators and five problem-focused nursing diagnoses, including the discriminator Cardiac pain and the diagnosis Acute pain. Three discriminators were associated with four risk nursing diagnoses, among these Acute neurological deficit with the diagnosis Risk of ineffective cerebral tissue perfusion. Conclusion: Significant associations were found between Manchester Triage System discriminators and the nursing diagnoses most frequently established in the emergency department.

Keywords: Emergency nursing. Triage. Nursing process. Nursing diagnosis. Nursing care. Terminology.

\section{RESUMEN}

Objetivo: Analizar asociaciones entre los discernidores del Sistema Triaje de Manchester y los Diagnósticos de Enfermería en pacientes adultos con prioridad clínica I (emergencia) y II (muy urgente).

Método: Estudio transversal realizado en la unidad de emergencia del sur de Brasil, entre abril y agosto de 2014, con la muestra de 219 pacientes. La colecta de datos fue realizada en el prontuario online de los pacientes. El análisis estadístico fue realizado con el uso del Test Exacto de Fisher o chi-cuadrado.

Resultados: Fue identificado el uso de 16 discernidores y 14 diagnósticos de enfermería. Hubo una asociación entre siete discernidores y cinco diagnósticos de enfermería del tipo foco en el problema, entre estos Dolor precordial o cardíaca y Dolor agudo. También hubo asociación entre tres discernidores y cuatro diagnósticos de enfermería de riesgo, entre estos Déficit neurológico agudo con el diagnóstico Riesgo de perfusión tisular cerebral ineficaz.

Conclusión: Existen asociaciones significativas entre los discernidores del Sistema Triaje de Manchester y los diagnósticos de enfermería más frecuente establecidos en la Unidad de Emergencia.

Palabras clave: Enfermería de urgência. Triaje. Proceso de enfermería. Diagnóstico de enfermería. Atención de enfermería. Terminología. 


\section{— INTRODUÇÃO}

O Sistema de Triagem de Manchester (STM) foi criado em 1994 em Manchester, Reino Unido, sendo utilizado como um protocolo válido, seguro e confiável em unidades de emergência de diversos países do mundo, entre eles o Brasil(1-2). O STM está estruturado em fluxogramas e discriminadores. Os fluxogramas representam a queixa principal do paciente, com base em sinais e sintomas, que levam à discriminação da prioridade clínica para o seu atendimento. Cada nível de prioridade aponta para um nível de gravidade, representado por uma cor e com indicação de um prazo máximo para o atendimento ${ }^{(3)}$. A prioridade clínica I corresponde ao nível de gravidade "emergência", é representada pela cor vermelha e o atendimento deve ser imediato; a prioridade clínica II corresponde ao nível de gravidade "muito urgente", é representada pela cor laranja e o atendimento deve ocorrer em até 10 minutos; a prioridade clínica III corresponde ao nível de gravidade "urgente", é representada pela cor amarela e o atendimento deve ocorrer em até 60 minutos; a prioridade clínica IV corresponde ao nível de gravidade "pouco urgente", é representada pela cor verde e o atendimento deve ocorrer em até 120 minutose, por último, a prioridade clínica $V$ corresponde ao nível de gravidade "não urgente", é representada pela cor azul e o atendimento pode ser em até 240 minutos $^{(3)}$. Para atribuir a prioridade clínica, o enfermeiro identifica junto ao paciente e/ou familiar o motivo que o trouxe ao atendimento e realiza a avaliação do estado de saúde do mesmo, com coleta de dados e exame físico focado na história clínica, de modo a selecionar o fluxograma e o discriminador do STM ${ }^{(4)}$.

Similarmente à avaliação do paciente pelo STM, o Processo de Enfermagem (PE) também guia o enfermeiro para o julgamento clínico e a tomada de decisão com base na coleta de dados do paciente (anamnese e o exame físico), com vistas a estabelecer o diagnóstico de enfermagem (DE)(5).

Com base nessa premissa, o enfermeiro ao realizar a classificação de risco pelo STM nas unidades de emergência, ainda que de maneira rápida e sucinta devido à característica desse protocolo, também utiliza etapas do PE, pois necessita de uma coleta de dados com identificação de sinais, sintomas e fatores de risco apresentados pelo paciente, para pensar criticamente sobre cada situação. Nesse sentido, pesquisa realizada em uma unidade de emergência brasileira com objetivo de identificar os possíveis DEs em pacientes classificados pelo STM nas prioridades clínicas I e II, concluiu que o protocolo pode favorecer a identificação de características definidoras, fatores de risco e fatores relacionados, subsidiando assim, o estabelecimento de DEs ${ }^{(6)}$. Todavia, os DEs elencados nesse estudo não foram realizados em ambiente real de cuidado, mas descritos como possíveis de serem elencados com base na revisão de prontuários.

Assim, desconhecem-se estudos publicados que tenham objetivado verificar associações entre os discriminadores do STM e os DEs, os quais poderão contribuir com evidências para o aprimoramento do julgamento clínico e tomada de decisão da enfermagem na prática clínica em urgência e emergência. Diante disso, e aliado à hipótese de que o STM oferece ao enfermeiro a oportunidade de identificar características definidoras, fatores relacionados e fatores de risco que possibilitam a seleção de DEs, entende-se como importante a realização do presente estudo que buscou analisar associações entre os discriminadores dos fluxogramas do STM e os DEs segundo a taxonomia da NANDA-I em pacientes adultos classificados com prioridade clínica I (emergência) e II (muito urgente) em uma emergência.

\section{MÉTODOS}

Estudo transversal realizado na Unidade de Emergência (UE) de um hospital universitário de grande porte do Sul do Brasil, certificado pela Joint Commission International' . A população do estudo foi constituída dos prontuários de pacientes adultos atendidos na UE durante o período de abril a agosto de 2014. Para o cálculo do tamanho da amostra, considerou-se o número de 503 atendimentos realizados no período de janeiro a abril de 2013 em pacientes com prioridade clínica I (emergência) e II (muito urgente), respeitando-se as proporções de cada uma das categorias, sendo $0,6 \%$ de pacientes com prioridade clínica I e 3,9\% de pacientes com a prioridade clinica II, assumindo-se uma margem de erro de $5 \%$ e um intervalo de confiança de 95\%. Assim, encontrou-se uma amostra de 219 pacientes, sendo 66 pacientes com prioridade clínica I e 153 pacientes com prioridade clínica II.

Os critérios de inclusão foram: prontuários de pacientes adultos classificados com prioridade clínica I (emergência) e II (muito urgente), com DEs elencados em prontuário online nas primeiras 24 horas. Os critérios de exclusão foram: prontuários de pacientes atendidos na UE por uma das pesquisadoras que era enfermeira do local; prontuários de pacientes que chegaram na UE por meio de contato prévio com a equipe, visto terem avaliação prévia à triagem e, muitas vezes, um pré-diagnóstico; prontuários de pacientes com mais de um atendimento no período da coleta, para evitar duplicidade de dados.

A coleta de dados foi realizada no prontuário online dos pacientes durante os meses de abril a agosto de 2014. Foram considerados os registros realizados no momento da 
classificação de risco do paciente com base no STM e no julgamento clínico do enfermeiro, bem como os diagnósticos de enfermagem que foram estabelecidos no decorrer do atendimento. Salienta-se, que tanto o STM como os diagnósticos de enfermagem adotam linguagem padronizada, o que diminui o viés de interpretação do registro.

O instrumento de coleta de dados continha dados sociodemográficos, clínicos, principais queixas, fluxograma e discriminador do STM, e os DEs elencados pelos enfermeiros na sequência do atendimento do paciente na UE, com suas respectivas características definidoras (sinais e sintomas) e/ou fatores de risco. Destaca-se que o $\mathrm{DE}$, bem como todas as demais etapas do PE, integra o prontuário do paciente neste hospital de forma informatizada, sendo realizado diariamente a despeito das dificuldades existentes em uma UE. Os dados coletados foram digitados, codificados e armazenados com o uso do programa Excel for Windows. A análise dos dados foi realizada no Statistical Package for Social Sciences (SPSS), versão 21.0. O ponto de corte para determinar os discriminadores dos fluxogramas do STM e os DEs a serem associados foi determinado pela estatística descritiva, considerando-se a frequência de ocorrência dos mesmos, uma vez que não existem estudos prévios que apontem evidências sobre como essas associações poderiam ser iniciadas. Assim, identificou-se dez discriminadores, bem como cinco DEs com foco no problema e quatro DEs de risco mais frequentemente aplicados na UE. A partir disso, as associações foram determinadas pelo teste exato de Fisher (por se tratar de amostras pequenas e independentes) ou qui-quadrado, considerando-se o nível de significância de $5 \%(p<0,05)$, além da razão de prevalência (RP) estimada pela regressão de Poisson, para medir a força das associações encontradas.

A pesquisa foi aprovada pelo Comitê de ética e Pesquisa em Saúde da instituição ( $n^{\circ}$ 140145). Os pesquisadores se comprometeram com o caráter confidencial das informações coletadas em prontuário assinando o Termo de Compromisso com a Utilização de Dados.

\section{RESULTADOS}

Dentre os 219 pacientes do estudo classificados nas prioridades clínicas I e II do STM, 110 (50,2\%) eram do sexo masculino, com idade média de 62,3 $\pm 15,3$ anos. As morbidades mais prevalentes foram a hipertensão arterial sistêmica $(54,3 \%)$ e a diabetes mellitus (28,3\%), conforme a Tabela 1.

Tabela 1 - Características sociodemográficas e clínicas dos pacientes avaliados pelo STM com prioridade clínica I e II atendidos na UE. Porto Alegre, RS, Brasil, 2015

$\begin{array}{lc}\text { Variável } & \mathbf{n = 2 1 9} \\ \text { Idade (anos) }^{*} & 62,3 \pm 15,3 \\ \text { Sexo masculino }^{\dagger} & 110(50,2) \\ \text { Escolaridade }^{\dagger \ddagger} & 69(31,5) \\ \text { Ensino Fundamental incompleto } & 25(11,4) \\ \text { Ensino Médio completo } & 21(9,6) \\ \text { Ensino Fundamental completo } & 10(4,6) \\ \text { Analfabetos } & 5(2,3) \\ \text { Ensino Superior completo } & 3(1,4) \\ \text { Ensino Médio incompleto } & 2(0,9) \\ \text { Ensino Superior incompleto } & \\ \text { Morbidades } & \\ \text { Hipertensão arterial sistêmica } & 119(54,3) \\ \text { Diabetes mellitus } & 62(28,3) \\ \text { Histórico de Cardiopatia Isquêmica } & 44(20,1) \\ \text { Neoplasias } & 43(19,6) \\ \text { Doença Pulmonar Obstrutiva Crônica } & 35(16,0) \\ \text { Tabagismo } & 32(14,6) \\ \text { Acidente Vascular Cerebral } & 30(13,7)\end{array}$

Fonte: Dados da pesquisa, 2015.

*Média \pm desvio-padrão, †n (\%), $\neq$ Ausência de registro da escolaridade em 84 pacientes (38,4\%).

Nota: A maioria dos pacientes do estudo apresentou mais de uma morbidade. 
Os pacientes apresentaram 20 diferentes queixas para a busca do atendimento. Dentre essas, a dispneia (37\%) e a dor $(35,6 \%)$ foram as principais. Foram identificados 14 diferentes fluxogramas do STM. Os mais frequentes foram "Dispneia em adulto" (71 - 32.4\%), "Mal estar em adulto" (51 - 23.3\%) e "Dor torácica" (45 - 20.5\%). A partir deles foram identificados 16 discriminadores (Tabela 2).
Os enfermeiros da UE estabeleceram 14 DEs com foco no problema e nove DEs de risco (Tabela 3).

Na análise dos dez discriminadores do STM com os cinco DEs com foco no problema mais frequentemente identificados na UE, houve associação significativa entre sete dos discriminadores e os cinco DEs, o que foi evidenciado pela RP, que mede a força de associação entre as variáveis.

Tabela 2 - Discriminadores do STM utilizados no atendimento de pacientes com prioridade clínica I e II na UE. Porto Alegre, RS, Brasil, 2015

$\begin{array}{lc}\text { Discriminadores }^{*} & \mathbf{n = 2 1 9} \\ \text { Dor precordial ou cardíaca } & 41(18,7) \\ \text { Saturação de Oxigênio muito baixa } & 32(14,6) \\ \text { Respiração inadequada } & 31(14,2) \\ \text { Pulso anormal } & 25(11,4) \\ \text { Déficit neurológico agudo } & 20(9,1) \\ \text { Hipoglicemia } & 13(5,9) \\ \text { Choque } & 12(5,5) \\ \text { Alteração súbita da consciência } & 12(5,5) \\ \text { Dor intensa } & 10(4,6) \\ \text { Convulsionando } & 9(4,1) \\ \text { Vômito de sangue } & 4(1,8) \\ \text { Frases entrecortadas } & 3(1,4) \\ \text { Evacuação de sangue vivo ou escurecido } & 3(1,4) \\ \text { Exaustão } & 2(0,9) \\ \text { Outros } & 2(0,9)\end{array}$

Fonte: Dados da pesquisa, 2015.

*Números expressos em n (\%).

Tabela 3 - Diagnósticos de Enfermagem estabelecidos pelos enfermeiros de uma UE para pacientes com prioridade clínica I e II. Porto Alegre, RS, Brasil, 2015

\section{Diagnósticos de Enfermagem com Foco no Problema*}

Padrão respiratório ineficaz

Dor aguda

$49(22,3)$

Débito cardíaco diminuído

Conforto prejudicado

$12(5,4)$

Negligência unilateral

$10(4,5)$

Perfusão tissular ineficaz cardiopulmonar

Ventilação espontânea prejudicada

Confusão aguda

$6(2,7)$

Desobstrução ineficaz de vias aéreas

Dor crônica

Volume de líquidos deficiente

Troca de gases prejudicada

Nutrição desequilibrada: menos que as necessidades corporais

Eliminação urinária prejudicada 
Diagnósticos de Enfermagem de Risco*

Risco de perfusão tissular cerebral ineficaz

Risco de quedas

Risco de glicemia instável

Risco de sangramento

Risco de volume de líquidos deficiente

Risco para disfunção respiratória prejudicada

Risco de desequilíbrio do volume de líquidos

Fonte: Dados da pesquisa, 2015.

*Números expressos em n (\%).

Assim, os discriminadores "Dor precordial ou cardíaca" ( $p$ $<0.001$; RP 4.17; IC 95\%:2.68-6.47) e"Dor intensa" ( $p<0.001$; RP 3.49; IC 95\%: 2.49-4.89) se associaram significativamente com o DE "Dor aguda". Da mesma forma, "Dor precordial ou cardíaca" se associou com DE "Conforto prejudicado" ( $p=0.008$; RP 4.38; IC 95\%: 1.47-13). O discriminador "Saturação de Oxigênio $\left(\mathrm{SatO}_{2}\right)$ muito baixa" ( $\mathrm{p}<0,001$; RP 3,12; IC 95\%: 2,274,30) e "Respiração inadequada" ( $p$ < 0.001; RP 2.51; IC 95\%:
1.80-3.50) apresentaram associação significativa com o DE "Padrão respiratório ineficaz", enquanto que "Pulso anormal" se associou significativamente com o DE" Débito cardíaco diminuído" ( $p=0.030$, RP 3.40; IC 95\%: 1.13-10.4). Os discriminadores "Déficit neurológico agudo" ( $p<0.001$; RP 36.85; IC 95\%: 11-119) e "Alteração súbita da consciência" ( $p=0.024$; RP 4.91; IC 95\%: 1.24-19.4) foram associados significativamente ao DE "Negligência unilateral", conforme sintetizado na Figura 1.

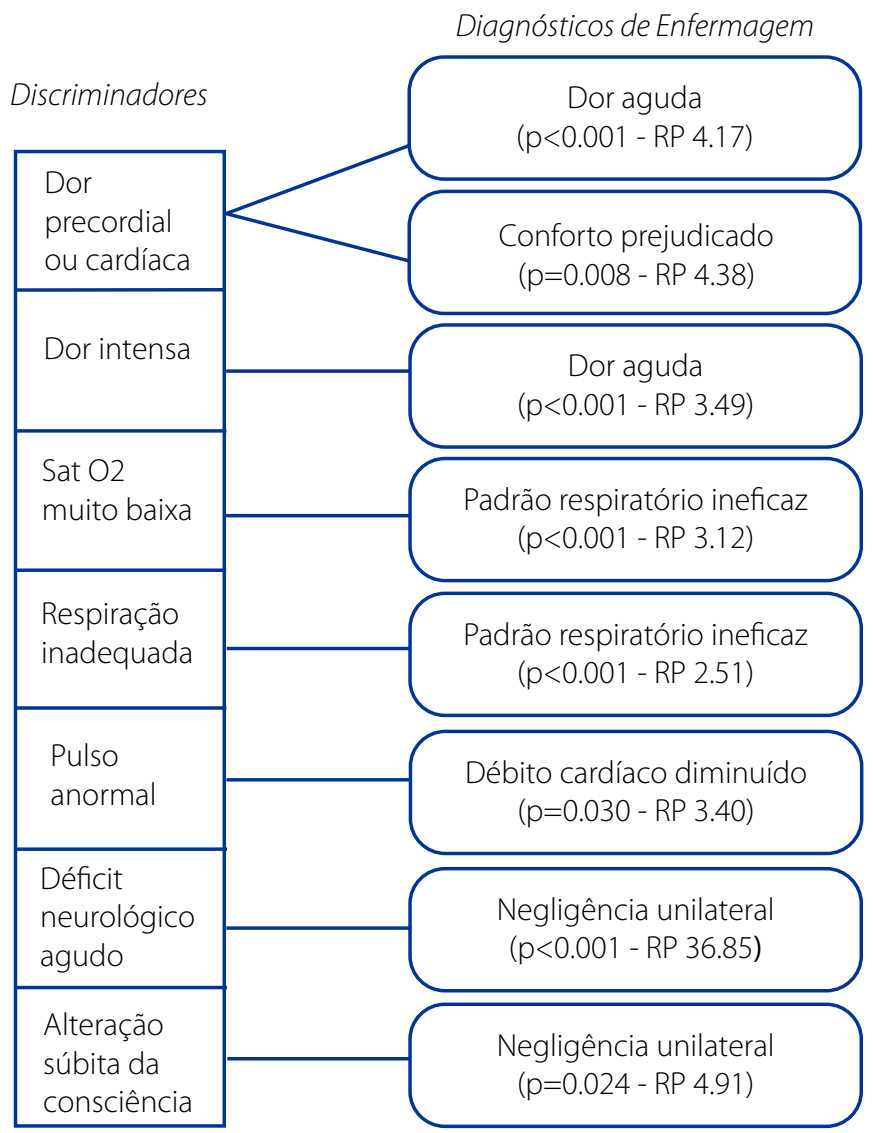

Figura 1 - Associações significativas entre os discriminadores do STM e os DEs com foco no problema elencados por enfermeiros de uma UE. Porto Alegre, RS, Brasil, 2015

Fonte: Dados da pesquisa, 2015. 
Também houve associação significativa entre três dos dez discriminadores do STM com os quatro DEs de risco mais frequentemente estabelecidos na UE.

Os discriminadores "Déficit neurológico agudo" $(p<0.001$; RP 4.13 e IC 95\%: 2.37-7.19) e "Convulsionando" ( $p=0.009 ;$ RP 2.31; IC 95\%: 1.23-4.34) apresentaram associação significativa com o DE "Risco de perfusão tissular cerebral ineficaz". O discriminador "Convulsionando" também se associou signi- ficativamente ao DE "Risco de quedas" ( $p=0.037$; RP 2.22; IC 95\%: 1.05-4.71). Por sua vez, o discriminador "Hipoglicemia" se associou significativamente ao DE "Risco de glicemia instável" ( $p<0.001$; RP 45.5; IC 95\%: 6.47- 319). Somente o discriminador "Choque" não apresentou associação significativa com os DEs. Da mesma forma, o DE "Risco de sangramento" não foi associado de forma significativa com os discriminadores. Esses resultados estão sintetizados na Figura 2.

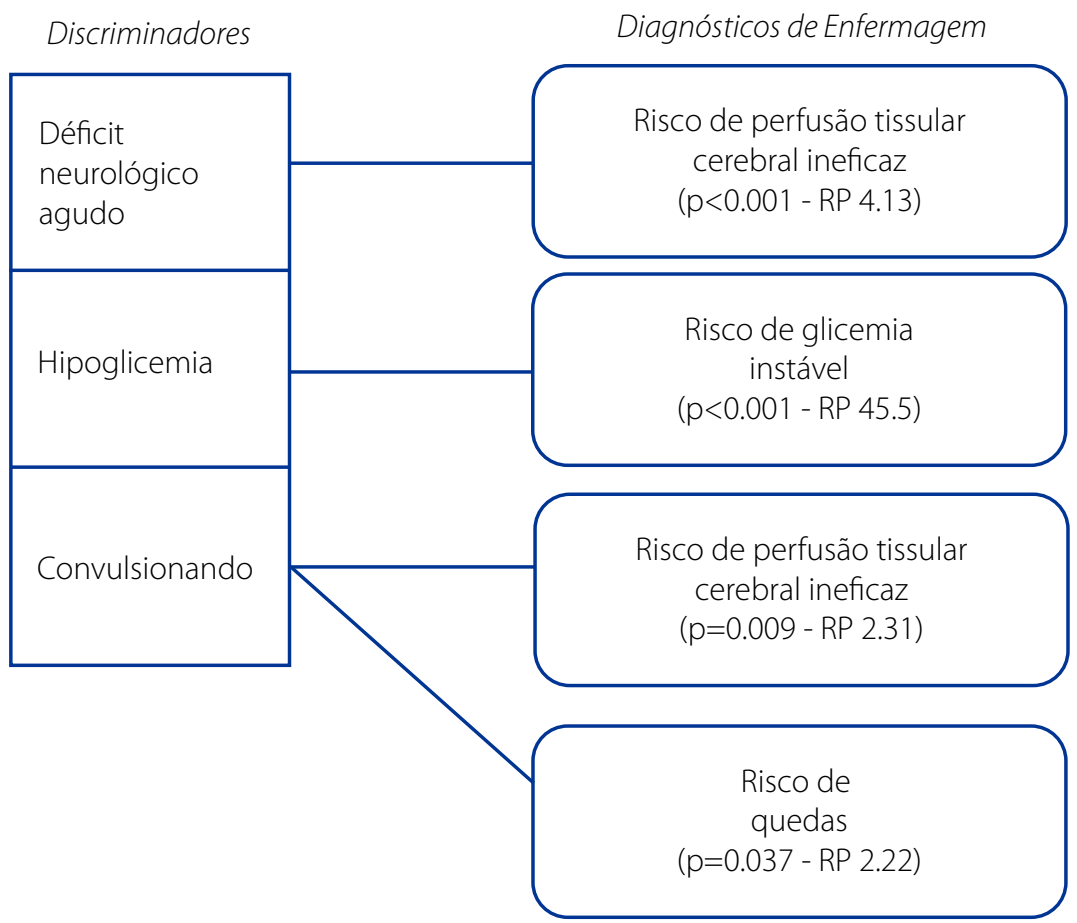

Figura 2 - Associações significativas entre os discriminadores do STM e os DEs de risco elencados por enfermeiros de uma UE. Porto Alegre, RS, Brasil, 2015.

Fonte: Dados da pesquisa, 2015

\section{DISCUSSÃO}

Os resultados desse estudo apontaram associações significativas entre os discriminadores dos fluxogramas do STM e os DEs mais frequentemente estabelecidos para pacientes de uma UE, demonstrando correspondência entre os mesmos e reforçando a ideia de que os dados coletados durante a classificação de risco também se constituem em pistas importantes para o estabelecimento do DE. Em ambos os processos (STM e DE) o enfermeiro realiza a avaliação do paciente com base na interpretação dos dados coletados de forma sistematizada, centrada na queixa principal e no exame físico do paciente. Esse processo favorece a identificação de características definidoras, fatores de risco e fatores relacionados, que embasam o raciocínio clínico, tanto para classificar a prioridade de atendimento do paciente como também para auxiliar a estabelecer o DE no decorrer do atendimento.

Nas associações encontradas, destacam-se as relacionadas às funções respiratória, cardíaca e neurológica; funções vitais ao funcionamento do organismo e fortemente tempo dependentes. Os discriminadores "Dor precordial ou cardíaca" e "Dor intensa"foram associados de forma significativa com o DE "Dor aguda", evidenciando que os primeiros que se constituem em fortes pistas para subsidiar o DE. Nota-se que as características definidoras do mesmo remetem aos discriminadores do STM citados, entre elas comportamento expressivo, evidência observada de dor, expressão facial, gestos protetores, relato codificado e relato verbal de dor ${ }^{(5)}$. Por sua vez, o discriminador "Dor precordial ou cardíaca" é definido como dor intensa em aper- 
to ou peso no meio do peito, que pode estar irradiando para o braço esquerdo ou pescoço, podendo associar-se à sudorese, náuseas, sensação de lipotimia e/ou dor epigástrica; - e o discriminador "Dor intensa" é definido como dor intolerável, insuportável, dilacerante, geralmente descrita como jamais sentida, de intensidade que varia entre 8-10 $0^{(3)}$. Também se identificou que os pacientes com o discriminador "Dor precordial ou cardíaca" possuem uma prevalência quatro vezes maior para o DE "Dor aguda", assim como aqueles com o discriminador "Dor intensa" apresentam uma prevalência três vezes maior para este $D E$, corroborando a hipótese desse estudo. Ao encontro disso, em pesquisa sobre a frequência dos DEs e suas características definidoras em pacientes com doenças cardiovasculares observou-se que o DE "Dor aguda" foi o mais prevalente $(70,7 \%)$, sendo a característica definidora de relato verbal de dor presente em $100 \%$ dos $\operatorname{casos}^{(7)}$.

O discriminador "Dor precordial ou cardíaca"também se associou significativamente ao DE "Conforto prejudicado", que apresenta entre suas características definidoras a ansiedade, choro, incapacidade de relaxar, inquietação, relato de falta de sentir-se à vontade com a situação, relato de sentir-se desconfortável e relato de sintomas de angústia ${ }^{(5)}$. A dor precordial geralmente está associada à Síndrome Coronariana $\mathrm{Aguda}^{(7)}$ e o enfermeiro deve estar atento à sua localização, característica, intensidade e duração, assim como aos fatores de risco, precipitantes e de alívio, para poder avaliar e atender adequadamente os pacientes na UE. Salienta-se ainda, que "Dor precordial ou cardíaca" é um discriminador do STM encontrado em diferentes fluxogramas, todavia, independentemente do fluxograma escolhido, este sempre levará o paciente ao nível de prioridade clínica II (muito urgente), o que pode justificar sua alta prevalência nesse e em outros estudos ${ }^{(8)}$. Além disso, os resultados também evidenciaram que os pacientes com o discriminador "Dor precordial ou cardíaca" apresentam uma prevalência quatro vezes maior de apresentar o DE "Conforto prejudicado", corroborando a alta frequência deste DE na UE. Assim, infere-se haver acurácia no estabelecimento desse DE, visto estar dentre as suas características definidoras a presença de sintomas de sofrimento e desconforto.

Outra manifestação clínica comum encontrada no estudo e corroborada pela literatura foi a dispnéia(9). Similarmente, estudo que analisou a demanda de pacientes classificados pelo STM encontrou entre os discriminadores mais frequentes o de "SatO ${ }_{2}$ muito baixa" e "Respiração inadequada". O discriminador "SatO, muito baixa" é definido por saturação de oxigênio menor que $95 \%$ em pacientes com terapia com $\mathrm{O}_{2}$ ou menor que $90 \%$ em ar ambiente, e o discriminador "Respiração inadequada" como paciente que não consegue respirar o suficiente para manter oxigenação adequada, podendo haver aumento do trabalho respiratório, sinais de respiração inadequada ou exaustão( ${ }^{(3)}$. Essas definições vêm ao encontro das características definidoras do DE "Padrão respiratório ineficaz" que são profundidade respiratória, batimento de asa de nariz, bradipneia, taquipneia, dispneia, uso da musculatura acessória para respirar e respiração com os lábios franzidos ${ }^{(5)}$. Nessa associação também se verificou que os pacientes com o discriminador "SatO ${ }_{2}$ muito baixa" possuem prevalência três vezes maior de apresentar o DE "Padrão respiratório ineficaz", e aqueles com o discriminador "Respiração inadequada" possuem prevalência duas vezes maior de apresentar esse mesmo DE, corroborando mais uma vez a ideia de que os discriminadores do STM podem subsidiar o raciocínio para o DE. Esse discriminador se encontra em diferentes fluxogramas do STM, uma vez que avalia o risco de vida dos pacientes e determina a prioridade clínica I (emergência).

Nesse contexto, estudo de validação de resultados de enfermagem da Classificação dos Resultados de Enfermagem (NOC) para o DE "Padrão respiratório ineficaz" apontou como prioritário o indicador Estado Respiratório: permeabilidade de vias aéreas ${ }^{(10-11)}$. Este achado corrobora os encontrados na presente investigação, uma vez que a permeabilidade das vias aéreas envolve a avaliação dos discriminadores "SatO 2 muito baixa" e "Respiração inadequada". Assim, ressalta-se que o enfermeiro deve estar atento aos sinais de desconforto ou de insuficiência respiratória para implementar precocemente intervenções que mantenham as vias aéreas do paciente desobstruídas.

Constatou-se também que os discriminadores "Déficit neurológico agudo", definido como qualquer perda da função neurológica nas últimas 24 horas, e "Alteração súbita da consciência", definido como a alteração da Escala de Coma de Glasgow nas últimas 12 horas em relação ao estado prévio(3), sustentam as características definidoras do DE "Negligência unilateral". Entre suas características definidoras estão a falha em movimentar o tronco e os membros no hemiespaço negligenciado, apesar de perceber um estímulo nesse espaço; falha em vestir o lado negligenciado, entre outras ${ }^{(5)}$. Essas associações significativas também demonstram que os pacientes triados com o discriminador "Déficit neurológico agudo" possuem uma prevalência 36 vezes maior de apresentar este $D E$, enquanto que os pacientes com o discriminador "Alteração súbita da consciência" apresentam uma prevalência quatro vezes maior para o mesmo DE. Cabe salientar que a instituição onde o estudo se desenvolveu é referência no atendimento a pacientes acometidos por acidente vascular cerebral (AVC) isquêmico, com equipe capacitada para evidenciar esses casos e 
tratá-los adequadamente, o que pode ter contribuído para o número de casos desse $D E$.

Outra associação significativa ocorreu entre o discriminador "Pulso anormal" e o DE "Débito cardíaco diminuído". Pulso anormal é definido como "bradicardia $(<60$ batimentos por minuto) ou taquicardia ( $>100$ batimentos por minuto) ou ainda um ritmo irregular"(3) O DE "Débito cardíaco diminuído" possui entre suas características definidoras elementos que se assemelham ao discriminador referido, como alterações no eletrocardiograma (ECG), bradicardia, palpitações e taquicardia(5). Ao encontro disso, estudo identificou a palpitação entre as principais características definidoras validadas para o DE "Débito cardíaco diminuído" em pacientes com insuficiência cardíaca descompensada ${ }^{(12)}$. Assim, o enfermeiro deve realizar o exame físico observando a frequência e o ritmo cardíaco, uma vez que o pulso anormal pode se manifestar com palpitações, síncope, dor precordial e até mesmo morte súbita. Um dos recursos utilizados para auxiliar o enfermeiro na detecção de arritmias é o ECG de 12 derivações, que deve ser realizado o mais breve possível. Essa associação também demonstrou que os pacientes com esse discriminador possuem uma prevalência três vezes maior de apresentar o DE "Débito cardíaco diminuído".

Outras associações significativas também foram evidenciadas entre os discriminadores do STM com os DEs de risco. "Déficit neurológico agudo"e "Convulsionando"foram associadas com o DE "Risco de perfusão tissular cerebral ineficaz". Este DE estabelece o risco de redução na circulação cerebra|(5), o que pode ser evidenciado por diferentes alterações neurológicas, incluindo as convulsões ${ }^{(3)}$. Estudo sobre a NOC validou 18 indicadores clínicos para avaliação de pacientes com o DE "Risco de perfusão tissular cerebral ineficaz"(13), dentre esses reflexos neurológicos prejudicados e nível reduzido de consciência, que se assemelham aos sinais avaliados pelo enfermeiro no uso do discriminador do STM "Déficit neurológico agudo". Destaca-se ainda, que os discriminadores "Déficit neurológico agudo" e "Alteração súbita da consciência" são frequentemente associados ao AVC isquêmico, condição clínica em que o paciente apresenta perfusão tissular cerebral ineficaz, e que representa uma das principais causas de morbimortalidade em todo o mundo ${ }^{(14)}$. Assim, o enfermeiro necessita reconhecer os fatores de risco, os sinais e sintomas deste agravo à saúde e atentar, principalmente, para o tempo do início dos mesmos, o que é fator determinante para a definição da prioridade clínica e prognóstico do paciente. Quanto mais precoce é o início do tratamento, maiores serão os benefícios terapêuticos. Também, identificou-se que os pacientes com "Déficit neurológico agudo" apresentam uma prevalência quatro vezes maior de apresentar o DE "Risco de perfusão tissular cerebral ineficaz", assim como os pacientes com o discriminador "Convulsionando" apresentaram uma prevalência duas vezes maior para esse DE. O discriminador "Convulsionando" também apresentou associação significativa com o DE "Risco de quedas", que possui dentre seus fatores de risco a alteração na função cognitiva, redução da força em extremidades inferiores ${ }^{(5)}$. Esses fatores vão ao encontro da situação clínica evidenciada pela convulsão e que traduz a vulnerabilidade para a queda. .

O discriminador "Hipoglicemia", definido como glicemia inferior a $55 \mathrm{mg} / \mathrm{dl}^{\prime \prime(3)}$ foi associado com o DE "Risco de glicemia instável". Esse DE se define pela "vulnerabilidade da variação dos níveis de glicose no sangue em relação aos parâmetros normais que pode comprometer a saúde $\mathrm{e}^{(5)} \mathrm{e}$ pode se manifestar por tremores, palidez, sonolência, confusão mental, dificuldade da coordenação motora e náuseas. Pacientes com esse discriminador, encontrado em diferentes fluxogramas do STM e que indica a classificação na prioridade clínica I (emergência), possuem prevalência 45 vezes maior de apresentar o DE "Risco de glicemia instável".

Dentre os dez discriminadores associados aos DEs com foco no problema se encontrou apenas um discriminador onde não houve associação, assim como entre as associações com os DEs de risco, apenas dois discriminadores não se associaram aos mesmos. Infere-se que essas associações não ocorreram por diferentes motivos, entre os quais, a possível mudança no estado de saúde do paciente em um curto período de tempo, avaliação inadequada com uso de discriminador e/ou um DE não acurado. Também podem ter ocorrido pelo fato de diferentes DEs apresentarem características definidoras muito semelhantes. Entretanto, mais estudos sobre a temática necessitariam ser realizados para responder essa questão.

Além dessa limitação, o estudo também não incluiu prontuários de pacientes pediátricos, em função de que existem fluxogramas específicos para essa população com sinais e sintomas peculiares a esta faixa etária, o que demandaria amostra diferente e um período de tempo maior para a realização do estudo.

Entretanto, o conjunto de discriminadores associados significativamente aos DEs dos pacientes adultos nesse estudo representam situações clínicas de alta prioridade para o cuidado de enfermagem, pois refletem a necessidade de suporte ao funcionamento físico, neurológico e homeostático, com potencial risco de vida. Corrobora-se que essas associações revelam que a utilização do STM, embora seja um protocolo para identificar a prioridade do atendimento do paciente, também favorece a identificação de características definidoras, fatores relacionados e de risco que susten- 
tam os DEs e, consequentemente, as intervenções a serem implementadas para se obter resultados positivos. Assim, entende-se que o uso das informações coletadas pelo STM, além de organizar a prioridade do atendimento do paciente na UE, também favorece o processo de raciocínio clínico que ocorre em todas as fases do processo de enfermagem, em que o enfermeiro reconhece pistas e evidências sobre a situação sob investigação, e a diferencia de outra similar para estabelecer o DE e/ou a intervenção mais acurada.

\section{— CONCLUSÃO}

Os resultados do estudo (oriundo de dissertação de mestrado apresentada ao Programa de Pós-graduação em Enfermagem da Escola de Enfermagem da Universidade Federal do Rio Grande do Su(15)) levam à conclusão de que existe associação significativa entre os discriminadores do STM e os DEs mais frequentemente estabelecidos na Unidade de Emergência. O discriminador Dor precordial ou cardíaca se associou aos DEs Dor Aguda e Conforto Prejudicado. O discriminador Dor intensa associou-se significativamente ao $\mathrm{DE}$ Dor aguda. Os discriminadores $\mathrm{SatO}_{2}$ muito baixa e Respiração inadequada associaram-se ao DE Padrão respiratório ineficaz. Pulso anormal associou-se ao DE Débito cardíaco diminuído, assim como Déficit neurológico agudo e Alteração súbita da consciência ao DE Negligência unilateral. Os discriminadores Déficit neurológico agudo e Convulsionando associaram se ao DE Risco de perfusão tissular cerebral ineficaz, Hipoglicemia ao DE Risco de glicemia instável, assim como o discriminador Convulsionando associou-se ao DE Risco de quedas.

Como fator limitador do estudo está o fato de ter sido realizado em uma única instituição, o que pode dificultar a generalização dos dados. Além disso, a ausência de estudos que abordem o STM e DEs nas áreas de urgência e emergência podem ter restringido a discussão dos achados. Ressalta-se a necessidade de outros estudos sobre os discriminadores do STM e os DEs em outros contextos assistenciais, a fim de buscar novas possibilidades de associações e fortalecer os resultados apresentados.

O estudo contribui para o aprofundamento do conhecimento de enfermagem na área de urgência e emergência, com dados que permitiram aproximar a teoria da prática, favorecendo as discussões sobre a temática e contribuindo para o desenvolvimento de habilidades para o julgamento clínico. Para a pesquisa de enfermagem, o estudo surgiu como uma nova possibilidade ou como subsídio para outras investigações, uma vez que foi o primeiro a ser realizado em ambiente clínico real envolvendo a temática sobre as possíveis associações entre os discriminadores do STM e os DEs da NANDA-I. Para a assistência, as associações testadas e que apontaram dados estatisticamente significativos favorecem o aprofundamento do conhecimento do enfermeiro e se mostram sob uma nova ótica para a busca da acurácia diagnóstica na emergência.

\section{口EFERÊNCIAS}

1. Azeredo TRM, Guedes HM, Almeida RAR, Chianca TCM, Martins JCA. Efficacy of the Manchester Triage System: a systematic review. Int Emerg Nurs. 2015[cited 2016 Jun 20];23(2):47-52. Available from: http://www.sciencedirect.com/science/article/pii/S1755599X14000512.

2. Santos AP, Freitas P, Martins HM. Manchester triage system version II and resource utilization in the emergency department. Emerg Med J. 2014[cited 2016 Jun 20];31(2):148-52. Available from: http://emj.bmj.com/content/ emermed/31/2/148.full.pdf

3. Mackway-Jones K, Marsden J, Windle J. Sistema Manchester de Classificação de Risco: classificação de risco na urgência e emergência. 1. ed. Belo Horizonte (MG): Grupo Brasileiro de Classificação de Risco; 2010.

4. Acosta AM, Duro CLM, Lima MADS. Activities of the nurse involved in triage/ risk classification assessment in emergency services: an integrative review. Rev Gaúcha Enferm. 2012[cited 2016 Jun 4];33(4):181-90. Available from: http:// www.scielo.br/pdf/rgenf/v33n4/en_23.pdf.

5. Heardman TH, Kamitsuru S. NANDA International Nursing Diagnoses: definitions \& classification, 2015-2017. 10th ed. Oxford: Wiley Blackwell; 2014.

6. Souza CCC, Mata LRF, Carvalho EC, Chianca TCM. Nursing diagnoses in patients classified as priority level I and II according to the Manchester protocol. Rev EsC Enferm USP. 2013[cited 2016 jun 26];47(6):1318-24. Available from: http:// www.scielo.br/pdf/reeusp/v47n6/en_0080-6234-reeusp-47-6-01318.pdf.

7. Pereira JMV, Cavalcanti ACD, Santana RF, Cassiano KM, Queluci GC, Guimarães, TCF. [Nursing diagnoses for inpatients with cardiovascular diseases]. EsC Anna Nery. 2011 [cited 2016 Jun 26];15(4):737-45. Available from: http://www.scielo.br/pdf/ean/v15n4/a12v15n4.pdf. Portuguese.

8. Christ M, Dorman H, Enk R, Popp S, Single S, Müller C, et al. Chest pain units or chest pain algorithm?. Med Klin Intensivmed Notfmed. 2014[cited 2016 Aug 4];109(7):495-503. Available from: https://link.springer.com/ article/10.1007\%2F-500063-013-0342-z.

9. Guedes HM, Almeida AGP, Ferreira F0, Vieira Júnior G, Chianca TCM. [Risk classification: portrait of a population using a Brazilian emergency service]. Rev Enf Ref. 2014[cited 2016 May 29];4(1):37-44. Available from: http://www.scielo, $\mathrm{mec} . \mathrm{pt} / \mathrm{pdf} / \mathrm{ref} / \mathrm{vserIVn} 1 / \mathrm{serl} V \mathrm{Vn} 1 \mathrm{a05}$.pdf. Portuguese.

10. Diniz AS, Silva AP, Souza CC, Chianca TCM. Clinical demand in an emergency care unit according to the Manchester triage system. Rev Eletrôn Enferm. 2014[cited 2016 Aug 26]; 16 (2):312-20. Disponível em: https://www.fen.ufg.br/fen_revista/v16/n2/pdf/v16n2a06.pdf.

11. Canto DF, Almeida MA. Nursing outcomes for ineffective breathing patterns and impaired spontaneous ventilation in intensive care. Rev Gaúcha Enferm. 2013[cited 2016 Aug 6];34(4):137-45. Available from: http://www.scielo.br/ pdf/rgenf//34n4/en_18.pdf.

12. Galvão PCC, Gomes ET, Figueirêdo TR, Bezerra SMMS. Diagnósticos de enfermagem aplicados a pacientes insuficiência cardíaca descompensada. Cogitare Enferm. 2016[citado 2017 mar 15];21(2):1-8. Disponível em: http://revistas. ufpr.br/cogitare/article/view/44646/28161. 
13. Almeida MA, Silva MB, Panato BP, Siqueira APO, Silva MP, Engelman B, et al. Clinical indicators to monitor patients with risk for ineffective cerebral tissue perfusion. Invest Educ Enferm. 2015[cited 2016 Aug 30];33(1):155-63. Available from: http://www.scielo.org.co/pdf/iee/v31n1/v33n1a18.pdf.

14. Gonzalez MM, Timerman S, Oliveira RG, Polastri TF, Dallan, LAP, Araújo S, et al. I Guideline for Cardiopulmonary Resuscitation and Emergency Cardiovascular Care - Brazilian Society of Cardiology: executive summary. Arq Bras Cardiol. 2013[cited 2016 Aug 3];100(2):105-13. Available from: http://www.scielo.br/ pdf/abc/v100n2/en_v100n2a01.pdf.
15. Franco B. Análise do Sistema de Triagem de Manchester como subsídio para 0 diagnóstico de enfermagem [dissertação]. Porto Alegre (RS): Escola de Enfermagem, Universidade Federal do Rio Grande do Sul; 2015 [citado 2016 mai 10]. Disponível em: http://www.lume.ufrgs.br/handle/10183/129598.

\section{AGRADECIMENTOS}

Este estudo foi apoiado pelo FIPE-HCPA (Fundo de Incentivo à Pesquisa e Eventos do Hospital de Clínicas de Porto Alegre).
Autor correspondente:

Amália de F. Lucena

E-mail: alucena@hcpa.edu.br
Recebido: 22.06.2017

Aprovado: 03.10.2017 